\title{
The Dark Side of the Propagators: exploring their analytic properties by a massive expansion
}

\author{
Fabio Siringo ${ }^{1, a}$ \\ ${ }^{1}$ Dipartimento di Fisica e Astronomia dell'Università di Catania, \\ INFN Sezione di Catania, Via S.Sofia 64, I-95123 Catania, Italy
}

\begin{abstract}
Analytical functions for the propagators of QCD, including a set of chiral quarks, are derived by a one-loop massive expansion in the Landau gauge, and are studied in Minkowski space, yielding a direct proof of positivity violation and confinement from first principles. Complex conjugated poles are found for the gluon propagator.
\end{abstract}

\section{Introduction}

Most of the non-perturbative approaches to QCD rely on numerical calculations in the Euclidean space, where a clear picture for the propagators of QCD emerges in Landau gauge by lattice simulations, by numerical solution of Schwinger-Dyson equations and, more recently, by unconventional variational methods[1-3].

However, since physics happens in Minkowski space, many important dynamical information cannot be extracted by the Euclidean formalism, unless we have an analytic function that can be continued to the physical space or the whole numerical analysis is carried out in Minkowski space[4]. Even the concept of a dynamical mass has no obvious meaning for confined particles like gluons and quarks. Thus, it is still questioned if the gluon propagator has poles, while some evidence of positivity violation has only been shown by indirect arguments.

Even if the analytic continuation of a limited set of data points is an ill-defined problem, a KällenLehmann spectral function was reconstructed in Ref.[5] from the lattice data of the gluon propagator, giving some direct evidence for positivity violation and the absence of any discrete mass pole on the physical real axis.

Quite recently, an analytical approach has been proposed that is based on a different expansion point for the exact Lagrangian of pure Yang-Mills theory in the Landau gauge[6, 7]. The new expansion is around a massive free-particle propagator, yielding a massive loop expansion with massive particles in the internal lines of the Feynman graphs. From first principles, without adding spurious counterterms or phenomenological parameters, at one-loop the expansion provides analytical universal functions for the dressing functions, predicting some scaling properties that are satisfied by the data of lattice simulations[8]. In the Euclidean space and Landau gauge, the massive expansion is in impressive agreement with the lattice data and the one-loop propagators are analytic functions that can be easily continued and studied in Minkowski space. Moreover, the massive expansion has been

ae-mail: fabio.siringo@ct.infn.it 
extended to full QCD by the inclusion of a set of chiral quarks in the Lagrangian[9] and the dynamical breaking of chiral symmetry is described on the same footing of gluon mass generation, providing a unified picture from first principles. Since analytic functions are derived for the one-loop propagators in the Landau gauge, they can be easily continued to Minkowski space where the spectral functions can be studied in detail. In this paper, a concise review is given of the main features of the propagators in Minkowski space, where their dark side emerges by the optimized massive expansion of Refs.[7, 9].

\section{The Optimized Massive Expansion}

The full Lagrangian of QCD, including $N_{f}$ massless chiral quarks, can be written as

$$
\mathcal{L}_{Q C D}=\mathcal{L}_{Y M}+\mathcal{L}_{f i x}+\mathcal{L}_{F P}+\mathcal{L}_{q}
$$

where $\mathcal{L}_{Y M}$ is the Yang-Mills term

$$
\mathcal{L}_{Y M}=-\frac{1}{2} \operatorname{Tr}\left(\hat{F}_{\mu \nu} \hat{F}^{\mu v}\right)
$$

$\mathcal{L}_{\text {fix }}$ is a covariant gauge fixing term, $\mathcal{L}_{F P}$ is the ghost Lagrangian arising from the Faddev-Popov determinant and $\mathcal{L}_{q}$ is the quark Lagrangian

$$
\mathcal{L}_{q}=\sum_{i=1}^{N_{f}} \bar{\Psi}_{i}\left[i \not \partial-g A_{a} \hat{T}_{a}\right] \Psi_{i} .
$$

The total action is $\mathcal{S}_{t o t}=\mathcal{S}_{0}+\mathcal{S}_{I}$, where the free-particle term $\mathcal{S}_{0}$ is the usual quadratic part that can be written in terms of the standard free-particle propagators of gluons, quarks and ghosts, namely $\Delta_{0}, S_{0}$ and $\mathcal{G}_{0}$, respectively.

As shown in Refs.[7, 9] a shift of the pole in the propagators can be introduced by an unconventional splitting of the total action. We may add and subtract the arbitrary terms $\delta \mathcal{S}_{g}, \delta \mathcal{S}_{q}$ in the total action

$$
\mathcal{S}_{0} \rightarrow \mathcal{S}_{0}+\delta \mathcal{S}_{q}+\delta \mathcal{S}_{g}, \quad \mathcal{S}_{I} \rightarrow \mathcal{S}_{I}-\delta \mathcal{S}_{q}-\delta \mathcal{S}_{g}
$$

and take

$$
\begin{aligned}
& \delta \mathcal{S}_{g}=\frac{1}{2} \int A_{a \mu}(x) \delta_{a b} \delta \Gamma_{g}^{\mu v}(x, y) A_{b v}(y) \mathrm{d}^{d} x \mathrm{~d}^{d} y \\
& \delta \mathcal{S}_{q}=\sum_{i=1}^{N_{f}} \int \bar{\Psi}_{i}(x) \delta \Gamma_{q}(x, y) \Psi_{i}(y) \mathrm{d}^{d} x \mathrm{~d}^{d} y
\end{aligned}
$$

where the vertex functions $\delta \Gamma_{g}, \delta \Gamma_{q}$ are given by a shift of the inverse propagators

$$
\begin{aligned}
\delta \Gamma_{g}^{\mu v}(x, y) & =\left[\Delta_{m}^{-1^{\mu v}}(x, y)-\Delta_{0}^{-1 \mu v}(x, y)\right] \\
\delta \Gamma_{q}(x, y) & =\left[S_{M}^{-1}(x, y)-S_{0}^{-1}(x, y)\right]
\end{aligned}
$$

and ${\Delta_{m}}^{\mu \nu}, S_{M}$ are massive free-particle propagators

$$
\begin{aligned}
& \Delta_{m}^{-1 \mu v}(p)=\Delta_{m}(p)^{-1} t^{\mu v}(p)+\frac{-p^{2}}{\xi} \ell^{\mu \nu}(p) \\
& \Delta_{m}(p)^{-1}=-p^{2}+m^{2}, \quad S_{M}(p)^{-1}=\not p-M .
\end{aligned}
$$


Here $t^{\mu \nu}, l^{\mu \nu}$ are Lorentz projectors and the masses $m$ and $M$ are totally arbitrary. Since $\delta \mathcal{S}_{q}$ and $\delta \mathcal{S}_{g}$ are added and subtracted again, the total action cannot depend on the masses, but any expansion in powers of the new shifted interaction $\mathcal{S}_{I} \rightarrow \mathcal{S}_{I}-\delta \mathcal{S}_{q}-\delta \mathcal{S}_{g}$ is going to depend on them at any finite order because of the truncation. Thus, while we are not changing the content of the theory, the emerging perturbative approximation is going to depend on the masses and can be optimized by a choice of $m$ and $M$ that minimizes the effects of higher orders, yielding a variational tool disguised to look like a perturbative method[7, 9]. The idea is not new and goes back to the works on the Gaussian effective potential[10-19] where an unknown mass parameter was inserted in the zeroth order propagator and subtracted from the interaction, yielding a pure variational approximation with the mass that acts as a variational parameter.

The shifts $\delta \mathcal{S}_{q}, \delta \mathcal{S}_{g}$ have two effects on the resulting perturbative expansion: the free-particle propagators are replaced by massive propagators and new two-point vertices are added to the interaction, arising from the counterterms that read

$$
\delta \Gamma_{g}^{\mu v}(p)=m^{2} t^{\mu v}(p), \quad \delta \Gamma_{q}(p)=-M .
$$

The Landau gauge is the optimal choice for the massive expansion[7] and from now on we will take the limit $\xi \rightarrow 0$. In Eq.(7) the gluon propagator becomes transverse and we can simplify the notation and drop the projectors $t^{\mu \nu}$ everywhere whenever each term is transverse. Moreover we drop all color indices in the diagonal matrices.

We can use the standard formalism of Feynman graphs with massive zeroth order propagators $\Delta_{m}$, $S_{M}$ and the counterterms $\delta \Gamma_{g}=m^{2}, \delta \Gamma_{q}=-M$ that must be added to the standard vertices of QCD.

Assuming that the effective coupling never reaches values that are too large[7], we may neglect higher loops and take a double expansion in powers of the total interaction and in powers of the coupling, retaining graphs with $n$ vertices at most and no more than $\ell$ loops.

The graphs contributing to the quark and ghost self-energy and to the gluon polarization are shown in Fig. 1 up to the third order and one-loop. Their calculation is straightforward and explicit analytical expressions are reported in Refs.[7, 9].

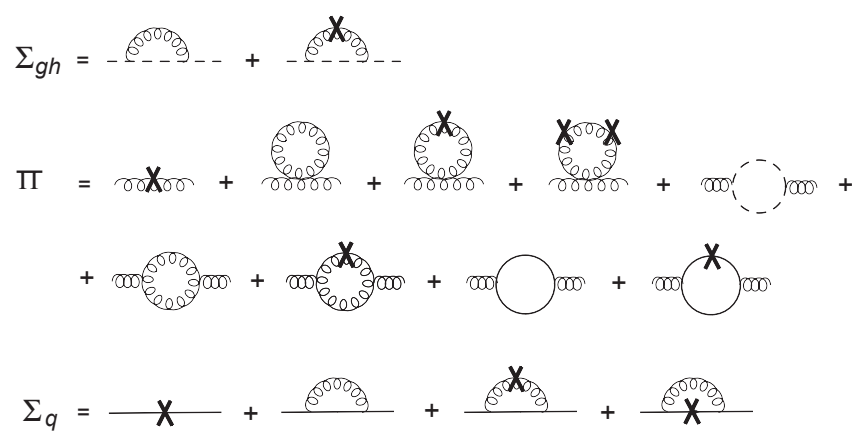

Figure 1. Two-point graphs with no more than three vertices and no more than one loop. The crosses are the counterterms $\delta \Gamma_{g}=m^{2}, \delta \Gamma_{q}=-M$. In this paper, the quark and ghost self energy and the gluon polarization are obtained by the sum of all the graphs in the figure. 


\section{Analytic continuation: pure Yang-Mills theory}

The dressed propagators of pure SU(N) Yang-Mills theory can be written as

$$
\Delta(p)^{-1}=-p^{2}+\frac{5}{8} \alpha m^{2}-[\Pi(p)-\Pi(0)], \quad \mathcal{G}(p)^{-1}=p^{2}-\Sigma_{g h}(p)
$$

where the ghost self-energy $\Sigma_{g h}$ and the gluon polarization $\Pi$ were evaluated in Ref.[7] as a sum of the graphs in Fig. 1 (omitting quark loops) and $\alpha$ is an effective coupling. The one-loop gluon and ghost propagators are made finite by standard wave function renormalization and explicit analytic expressions were derived by dimensional regularization in Ref.[7].

It is useful to introduce the adimensional ghost and gluon dressing functions

$$
\chi(p)=p^{2} \mathcal{G}(p), \quad J(p)=-p^{2} \Delta(p) .
$$

They can be written as

$$
[\alpha \chi(s)]^{-1}=G(s)+G_{0} \quad[\alpha J(s)]^{-1}=F(s)+F_{0}
$$

where $s=-p^{2} / m^{2}$ is the Euclidean momentum and the two adimensional functions $F(s), G(s)$ are given by the polarization and self energy graphs in Fig. 1, while all the constants are grouped together in the finite one-loop renormalization constants $F_{0}$ and $G_{0}$ that are the only free parameters to be optimized. Being equivalent to a variation of the subtraction point, any change of the additive constant can be seen as a variation of the renormalization scheme yielding a special case of optimized perturbation theory that has been proven to be very effective for the convergence of the expansion[20].

A very important consequence of Eq.(11) is that, up to an arbitrary multiplicative renormalization constant, the inverse dressing functions are given by the universal functions $F(s)$ and $G(s)$ up to an additive renormalization constant. Such scaling property is satisfied quite well by the lattice data

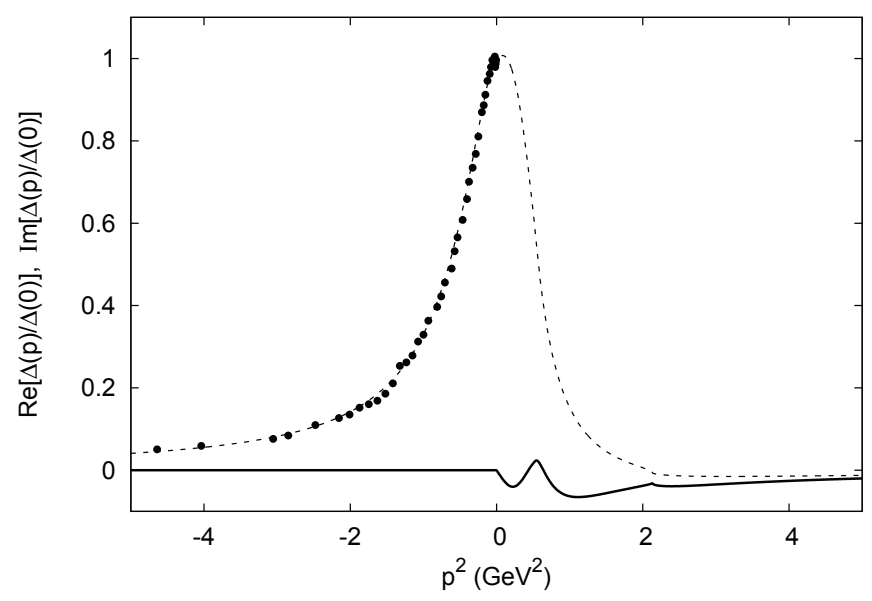

Figure 2. The real and the imaginary part of the gluon propagator are displayed together with the lattice data of Ref.[21] $(N=3, \beta=5.7, L=96)$. The propagator is normalized by its finite value at $p^{2}=0$ and is evaluated by Eq.(11) with the optimal choice $F_{0}=-1.05$ and $m=0.73 \mathrm{GeV}$. 


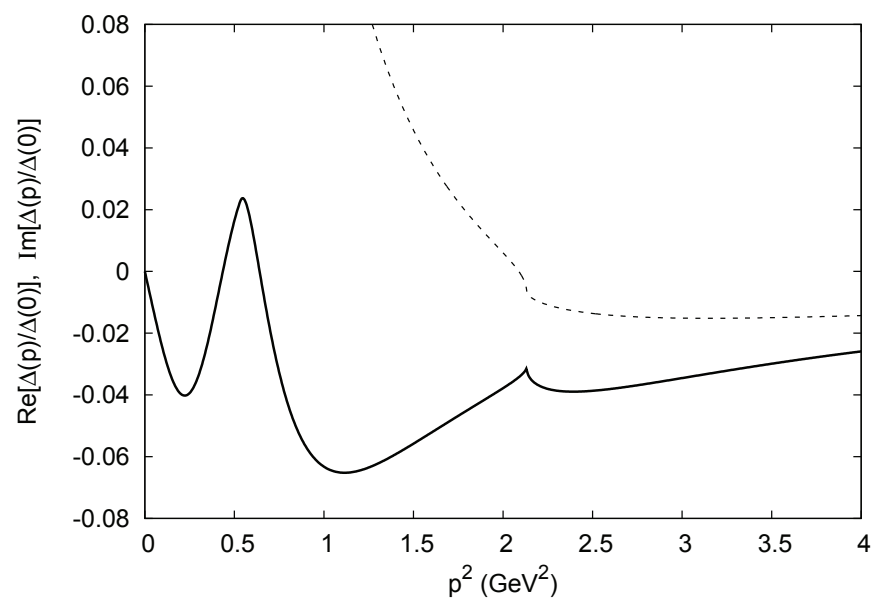

Figure 3. The real and the imaginary part of the gluon propagator (enlargement of Fig. 2).

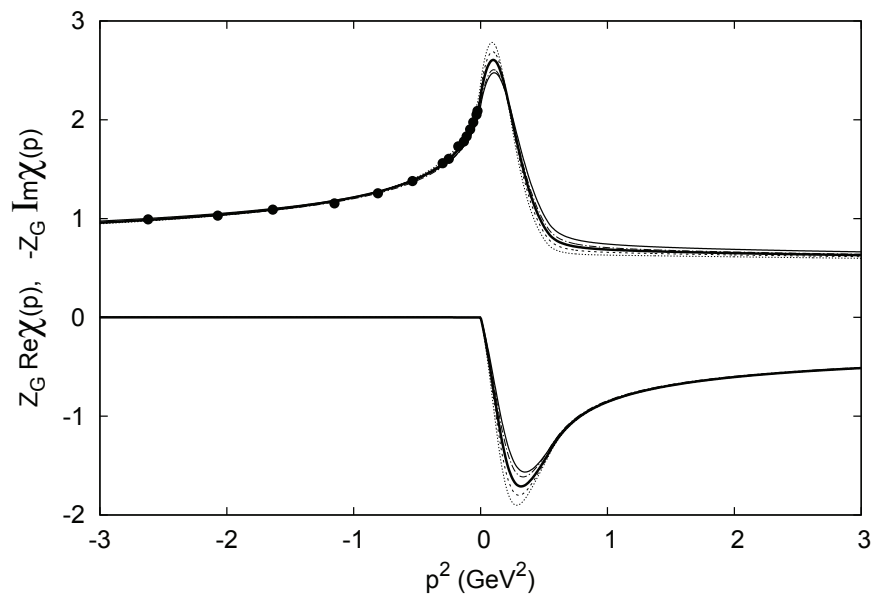

Figure 4. Real part $\operatorname{Re} \chi$ and imaginary part $-\operatorname{Im} \chi=\pi p^{2} \rho$ of the ghost dressing function according to Eq.(11) for $m=0.73 \mathrm{GeV}$ and several values of $G_{0}$ in the range $0.2<G_{0}<0.3$. The points are the lattice data of Ref.[21] ( $N=3, \beta=5.7, L=80$ ). The best agreement with the data points is obtained for $G_{0}=0.24$ (solid line). The dressing function is scaled by a finite renormalization constant $Z_{G}$.

for $\mathrm{SU}(2)$ and $\mathrm{SU}(3)$ that collapse on the same universal curves $F(s), G(s)$ in the infrared[6-9], thus confirming that higher order terms can be made negligible by an optimized choice of the constants $F_{0}, G_{0}$.

For $S U(3)$ and $-p^{2}<4 \mathrm{GeV}^{2}$ the lattice data of Ref.[21] are very well reproduced by setting $F_{0}=-1.05$ and $m=0.73 \mathrm{GeV}$ in Eq.(11). Some deviation occurs for $-p^{2}>4 \mathrm{GeV}^{2}$ because of the large logs that require a resummation by $\mathrm{RG}$ equations in the UV. 


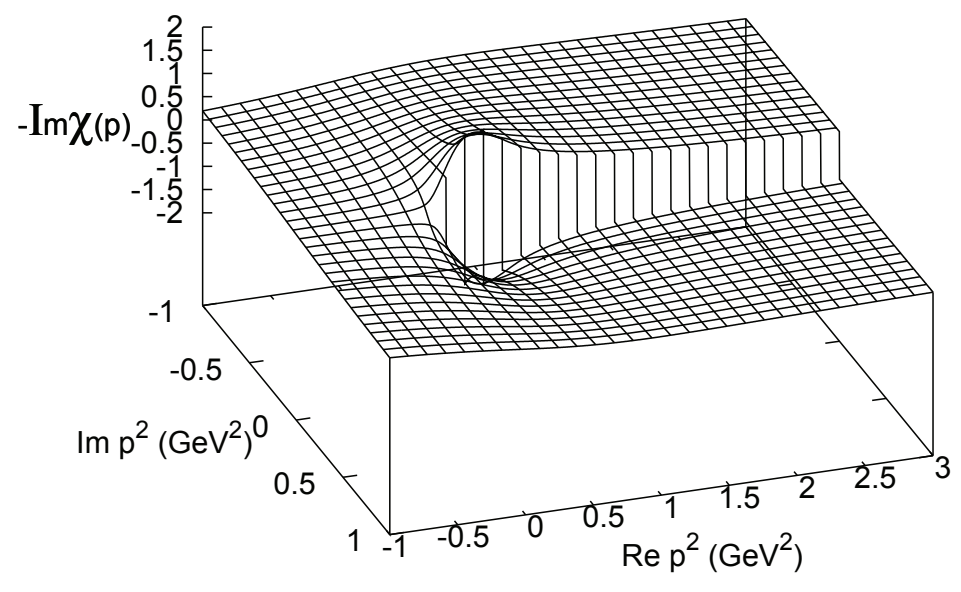

Figure 5. Imaginary part $-\operatorname{Im} \chi=\pi p^{2} \rho$ of the ghost dressing function in the complex plane for $m=0.73 \mathrm{GeV}$ and $G_{0}=0.24$

The gluon propagator can be continued to Minkowski space by setting $s=-p^{2} / m^{2}-i \varepsilon$ and the resulting complex function is shown in Fig. 2. The imaginary part has a cut for $p^{2}>0$ where it defines a spectral function. The lack of any sharp peak or pole on the real axis and the violation of positivity can be regarded as a direct proof of confinement.

Out of the real axis, in the complex plane, the propagator has two conjugated poles at $p^{2} \approx$ $(0.16 \pm 0.60 i) \mathrm{GeV}^{2}$, close to the imaginary axis, as predicted by the $i$-particle scenario[22] emerging from the refined version[23-25] of the Gribov-Zwanziger model[26].

The one-loop ghost propagator, by Eq.(11) mantains a pole at $p^{2}=0$. The analytic continuation $s=-p^{2} / m^{2}-i \varepsilon$ yields

$$
\begin{aligned}
& \operatorname{Re} \mathcal{G}\left(p^{2}+i \varepsilon\right)=\frac{\operatorname{Re} \chi\left(p^{2}\right)}{p^{2}} \\
& \operatorname{Im} \mathcal{G}\left(p^{2}+i \varepsilon\right)=\frac{\operatorname{Im} \chi\left(p^{2}\right)}{p^{2}}-\pi \chi(0) \delta\left(p^{2}\right)
\end{aligned}
$$

and we can define a spectral function on the cut

$$
\rho\left(p^{2}\right)=-\frac{1}{\pi} \operatorname{Im} \mathcal{G}\left(p^{2}+i \varepsilon\right)=\chi(0) \delta\left(p^{2}\right)-\frac{1}{\pi} \frac{\operatorname{Im} \chi\left(p^{2}\right)}{p^{2}}
$$

which has a continuous term given by the imaginary part of the dressing function divided by $-p^{2}$. The details of the continuous term of the spectral function are shown in Fig. 4 by the direct plot of $-\operatorname{Im} \chi$, together with the real part $\operatorname{Re} \chi$ and the lattice data of Ref.[21] $(N=3, \beta=5.7, L=80)$. We observe that the discrete and the continuous terms have opposite sign in Eq.(13), violating the positivity condition. In the Euclidean range $p^{2}<0$, the ghost dressing function is not too much 


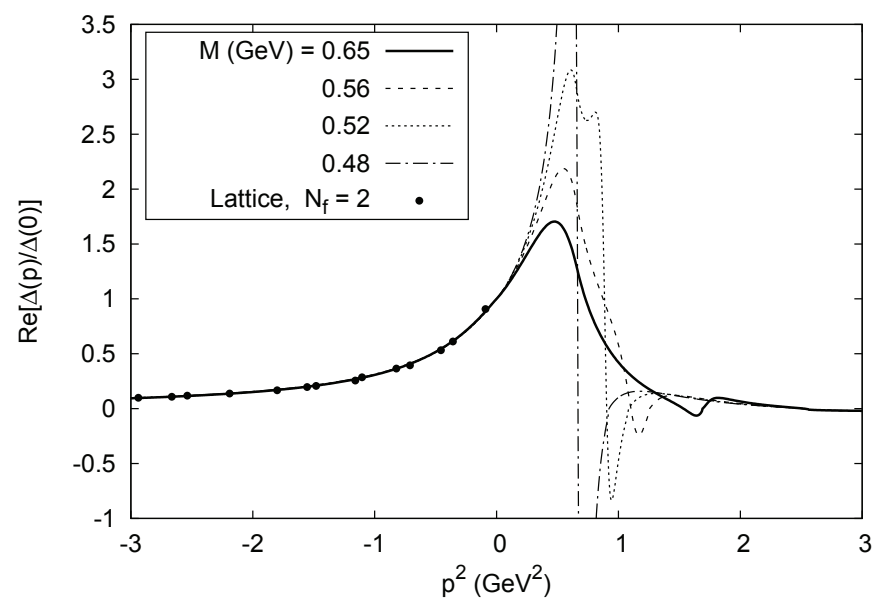

Figure 6. The real part of the gluon propagator is evaluated by setting $s=-p^{2} / m^{2}-i \varepsilon$, for $m=0.80 \mathrm{GeV}$ and several values of $M=0.48,0.52,0.56,0.65 \mathrm{GeV}$. The constant $F_{0}$ varies in the range $-0.65<F_{0}<-0.6$ in order to keep all curves on the lattice data in the Euclidean space, for $p^{2}<0$. The data points are extracted from Fig. 1 of Ref.[27] for $N_{f}=2$. The propagator is normalized by its finite value at $p^{2}=0$.

sensitive to a change of the additive constant $G_{0}$. In Fig. 4, a change of $G_{0}$ in the range $0.2<G_{0}<0.3$ is compensated by a change of the finite renormalization constant $Z_{G}$, so that $Z_{G} \chi\left(p^{2}\right)$ stays on the lattice data points. The best agreement is found for $G_{0}=0.24$ and is shown as a solid line in Fig. 4 . The imaginary part has a wide peak at $p^{2} \approx(0.56)^{2} \mathrm{GeV}^{2}$ and never changes sign. As shown in Fig. 5, it is finite in the whole complex plane, with a cut on the real axis where the spectral function is defined by Eq. (13).

\section{Analytic continuation: chiral QCD}

The inclusion of a set of chiral quarks requires the calculation of the quark loops contributing to the gluon polarization and the quark self-energy $\Sigma_{q}$ as shown in Fig. 1. Since there are no one-loop graphs with quark lines that contribute to the ghost self-energy $\Sigma_{g h}$, the one-loop ghost dressing function of QCD is the same of pure Yang-Mills theory.

The gluon polarization of the full theory is obtained from the result for pure Yang-Mills theory by just adding the quark loops of Fig. 1. Explicit analytical expressions are reported in Ref.[9].

The real part of the gluon propagator is shown in Fig. 6 for $s=-p^{2} / m^{2}-i \varepsilon$. While rather insensitive to the choice of $M$ in the Euclidean space, the shape of the propagator depends on $M$ when plotted as a function of the time-like momentum $p^{2}>0$. The data points in the figure are the lattice data of Ref.[27] for two light quarks, having no lattice data for the gluon propagator in the chiral limit. We observe the presence of a positive peak at $p^{2} \approx m^{2}$ and a negative peak just before the two-particle threshold $p^{2} \approx(2 M)^{2}$ where the real part of the propagator changes sign and becomes positive. As shown in Fig. 7, where the imaginary part of the propagator is displayed, the spectral density becomes negative and its positivity violation is a direct proof of confinement. At variance with pure Yang-Mills theory, there is a two-particle threshold at $p^{2} \approx(2 M)^{2}$ where the spectral function turns positive for a while. Besides being more rich on the real positive axis $p^{2}>0$, for $N_{f}=2$ the unquenched gluon 


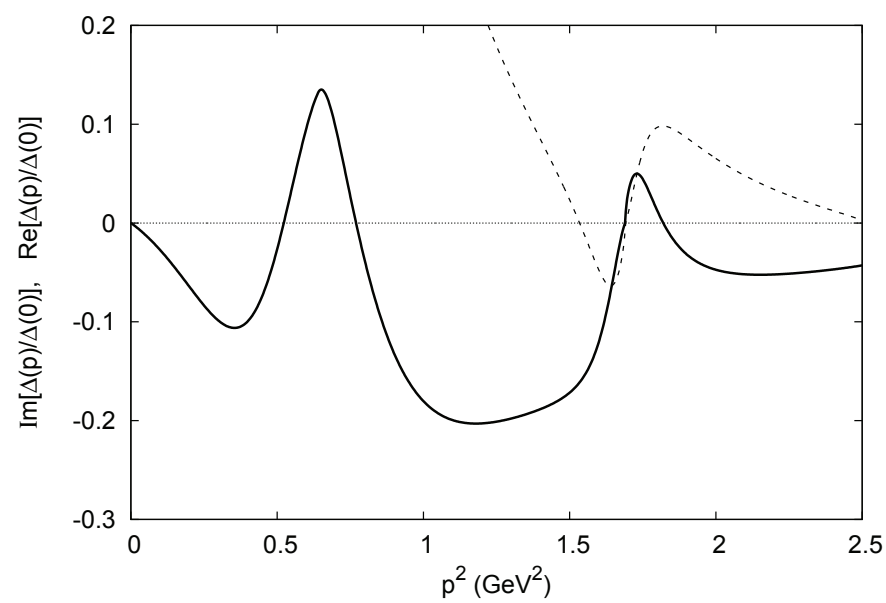

Figure 7. The imaginary part of the propagator is evaluated by setting $s=-p^{2} / m^{2}-i \varepsilon$ for the optimal set $m=0.80 \mathrm{GeV}, M=0.65 \mathrm{GeV}, F_{0}=-0.65$ (solid line). The dashed line is a detail of the real part. The propagator is normalized by its finite value at $p^{2}=0$.

propagator has more poles in the complex plane. For the optimal set $m=0.8 \mathrm{GeV}, M=0.65 \mathrm{GeV} \mathrm{We}$ find two pairs of conjugated poles at $p^{2} \approx(1.69, \pm 0.1) \mathrm{GeV}^{2}$ and $p^{2} \approx(0.54, \pm 0.52) \mathrm{GeV}^{2}$.

The quark self energy $\Sigma_{q}$ is evaluated by the tree term $\delta \Gamma_{q}=-M$ and the three one-loop graphs in Fig. 1. In the dressed quark propagator $S(p)$ the mass $M$ is canceled by the tree term $\delta \Gamma_{q}=-M$. However, even in the chiral limit, a mass function is generated for the quarks by the interaction terms[9].

The dressed quark propagator can be written as

$$
S(p)=S_{p}\left(p^{2}\right) \not p+S_{M}\left(p^{2}\right)
$$

where the scalar functions $S_{p}, S_{M}$ follow from the one-loop self energy, yielding explicit analytical expressions that can be easily continued to Minkowski space by setting $s=-p^{2} / m^{2}-i \varepsilon$. The imaginary parts have a cut on the real positive axis $p^{2}>0$ where we can define two spectral densities

$$
\begin{aligned}
\rho_{M}\left(p^{2}\right) & =-\frac{1}{\pi} \operatorname{Im} S_{M}\left(p^{2}\right) \\
\rho_{p}\left(p^{2}\right) & =-\frac{1}{\pi} \operatorname{Im} S_{p}\left(p^{2}\right)
\end{aligned}
$$

so that the propagator reads

$$
S(p)=\int_{0}^{\infty} \mathrm{d} q^{2} \frac{\rho_{p}\left(q^{2}\right) \not p+\rho_{M}\left(q^{2}\right)}{p^{2}-q^{2}+i \varepsilon} .
$$

Any observable fermion must satisfy the positivity conditions

$$
\begin{gathered}
\rho_{p}\left(p^{2}\right) \geq 0 \\
p \rho_{p}\left(p^{2}\right)-\rho_{M}\left(p^{2}\right) \geq 0
\end{gathered}
$$




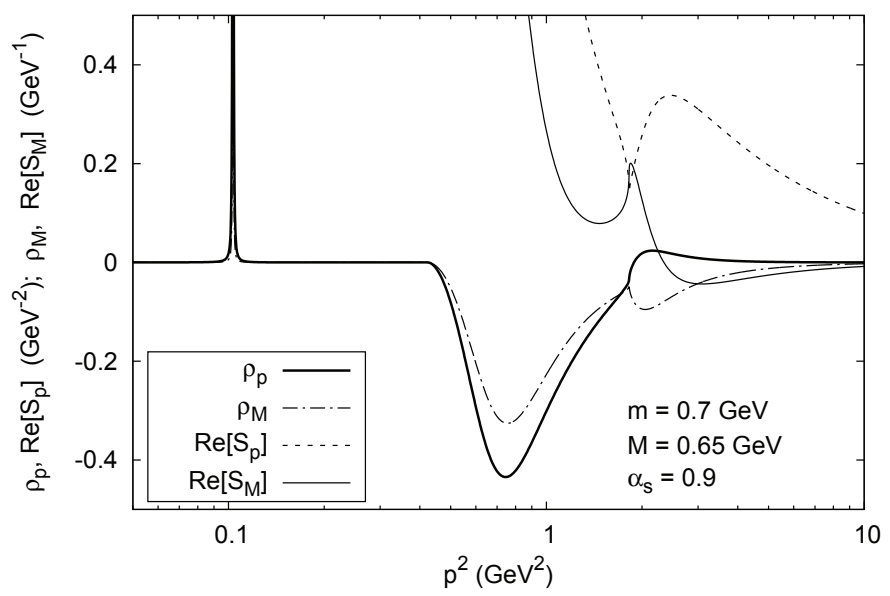

Figure 8. Details of the quark spectral functions for $\alpha_{s}=0.9, M=0.65 \mathrm{GeV}, m=0.7 \mathrm{GeV}$.

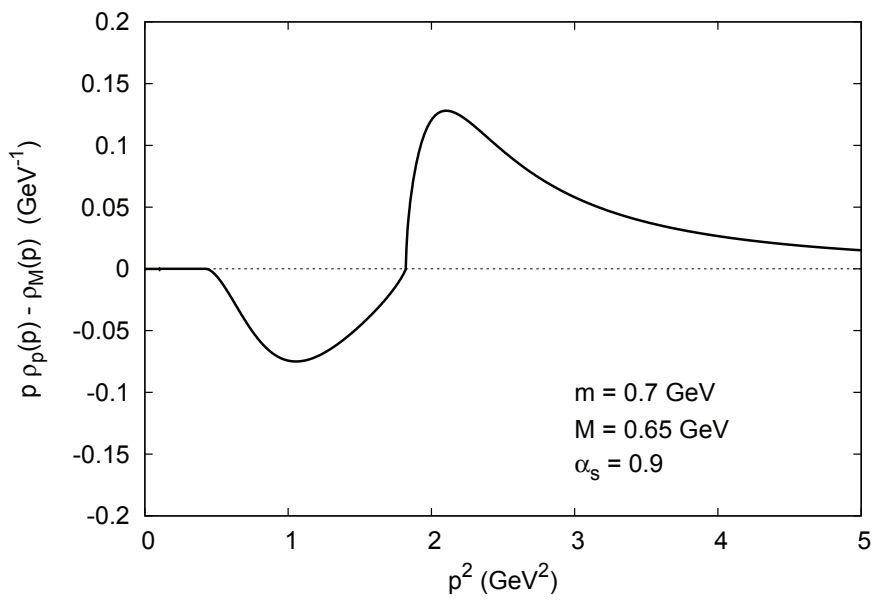

Figure 9. The quark spectral function $\left[p \rho_{p}\left(p^{2}\right)-\rho_{M}\left(p^{2}\right)\right]$ is shown as a function of the physical momentum $p^{2}=-p_{E}^{2}$ for $m=0.7 \mathrm{GeV}, \alpha_{s}=0.9$ and $M=0.65 \mathrm{GeV}$. The positivity condition of Eq.(18) is violated for $p^{2}>q_{1}^{2} \approx M^{2}$ below the two-particle threshold $q_{2}^{2} \approx(m+M)^{2}$.

that are strongly violated by the quark propagator, yielding a direct proof of confinement.

The spectral functions are shown in Figs. 8, 9 for $\alpha_{s}=0.9, M=0.65 \mathrm{GeV}$. We recognize a discrete term at $p \approx 0.32 \mathrm{GeV}$, that arises from the pole of the propagator. We can identify two different thresholds. A first threshold $q_{1}^{2} \approx M^{2}$ at the onset of a negative continuum spectral density $\left(q_{1}^{2} \approx(0.65)^{2} \approx 0.42 \mathrm{GeV}^{2}\right.$ in Figs. 8,9$)$. A second threshold $q_{2}^{2} \approx(M+m)^{2}$ where the spectral density turns positive $\left(q_{2}^{2} \approx(1.35)^{2} \approx 1.82 \mathrm{GeV}^{2}\right.$ in Figs. 8, 9). While this second threshold can be identified with the usual two-particle threshold and the high-energy states have a positive spectral density above $q_{2} \approx(m+M)$, the negative spectral density above $q_{1} \approx M$ has no obvious physical meaning. It 
violates the positivity condition (17) and cannot be related to any kind of free-particle behavior. Thus the quark propagator can only describe confined particles. No complex poles are observed for the quark propagator.

\section{Concluding remarks}

The massive expansion that was developed for pure Yang-Mills theory in Refs.[6, 7] and extended to full QCD in Ref.[9] has been reviewed and used as a tool for exploring the dark side of the propagators in Minkowski space.

By a direct comparison with the lattice data, the expansion is optimized in the Euclidean space yielding accurate analytic propagators that can be easily continued to Minkowski space. Thus the method provides a powerful tool for the study of dynamical properties and spectral functions that can be hardly extracted from any numerical data set. From this point of view, the massive expansion is very predictive and gives a direct proof of positivity violation and confinement for all the particles involved.

While no direct dynamical content can be given to the gluon mass and to the mass parameters, the discrete one-particle term in the quark spectral functions can be identified as the (confined) physical mass of the constituent quarks. On the other hand, the mass parameters $m, M$ are strongly related to the thresholds of the spectral functions and determine their rich behavior that is observed in Minkowski space.

\section{References}

[1] F. Siringo and L. Marotta, Eur. Phys. J. C 44, 293 (2005).

[2] F. Siringo, Mod. Phys. Lett. A 29, 1450026 (2014), [arXiv:1308.4037].

[3] F. Siringo, Phys. Rev. D 88, 056020 (2013); Phys. Rev. D 89, 025005 (2014); Phys. Rev. D 90, 094021 (2014); Phys. Rev. D 92, 074034 (2015); arXiv:1507.05543.

[4] S. Strauss, C. S. Fischer, C. Kellermann, Phys. Rev. Lett. 109, 252001 (2012).

[5] D. Dudal, O. Oliveira, P. J. Silva, Phys. Rev. D 89, 014010 (2014).

[6] F. Siringo, arXiv:1509.05891.

[7] F. Siringo, Nucl. Phys. B 907, 572 (2016), [arXiv:1511.01015].

[8] F.Siringo, arXiv:1607.02040.

[9] F. Siringo, arXiv:1605.07357.

[10] P.M. Stevenson, Phys. Rev. D 32, 1389 (1985).

[11] F. Siringo, L. Marotta, Phys. Rev. D 78, 016003 (2008); Phys. Rev. D 74, 115001 (2006).

[12] F. Siringo, Phys. Rev. D 86, 076016 (2012), [arXiv: 1208.3592v2].

[13] I. Stancu and P. M. Stevenson, Phys. Rev. D 42, 2710 (1990).

[14] I. Stancu, Phys. Rev. D 43, 1283 (1991).

[15] M. Camarda, G.G.N. Angilella, R. Pucci, F. Siringo, Eur. Phys. J. B 33, 273 (2003).

[16] L. Marotta, M. Camarda, G.G.N. Angilella and F. Siringo, Phys. Rev. B 73, 104517 (2006).

[17] L. Marotta and F. Siringo, Mod. Phys. Lett. B, 26, 1250130 (2012).

[18] F. Siringo, Phys. Rev. D 62, 116009 (2000); Europhys. Lett. 59, 820 (2002).

[19] F. Siringo and L. Marotta, Int. J. Mod. Phys. A 25, 5865 (2010).

[20] P.M. Stevenson, Nucl. Phys. B 868, 38 (2013); Nucl. Phys. B 910, 469 (2016).

[21] I.L. Bogolubsky, E.M. Ilgenfritz, M. Muller-Preussker, A. Sternbeckc, Phys. Lett. B 676, 69 (2009). 
[22] L. Baulieu, D. Dudal, M. S. Guimaraes, M. Q. Huber, S. P. Sorella, N. Vandersickel, D. Zwanziger, Phys.Rev.D 82, 025021 (2010).

[23] D. Dudal, J. A. Gracey, S. P. Sorella, N. Vandersickel, H. Verschelde, Phys. Rev. D 78, 065047 (2008).

[24] D.Dudal, S.P.Sorella, N.Vandersickel, H.Verschelde, Phys. Rev. D 77, 071501 (2008).

[25] D. Dudal, S. P. Sorella, N. Vandersickel, Phys. Rev. D 84, 065039 (2011).

[26] D. Zwanziger, Nucl. Phys. B 323, 513 (1989).

[27] A. Ayala, A. Bashir, D. Binosi, M. Cristoforetti and J. Rodriguez-Quintero, Phys. Rev. D 86, 074512 (2012). 